\title{
Long non-coding RNA MIAT competitively binds miR-150-5p to regulate ZEB1 expression in osteosarcoma
}

\author{
HAO JIN, XIN JIN, WEIGUANG CHAI, ZHIQIANG YIN, YANG LI, FENG DONG and WENBO WANG \\ Department of Orthopaedics, The First Affiliated Hospital of Harbin Medical University, \\ Harbin, Heilongjiang 150006, P.R. China
}

Received September 4, 2016; Accepted June 19, 2018

DOI: $10.3892 /$ ol.2018.9671

\begin{abstract}
Long non-coding RNAs (LncRNAs), are significant in a number of biological stages and illnesses. The myocardial infarction associated transcript (MIAT) serves a function in numerous types of illness and physiological and pathological processes, including paranoid schizophrenia, diabetic retinopathy, myocardial infarction and neuroendocrine prostate cancer. However, the function of the lncRNA MIAT in the development of osteosarcoma is unknown. It has been identified that during the development of osteosarcoma, MIAT is upregulated in tumor tissues compared to adjacent non-tumor tissues. The spreading and proliferation of osteosarcoma cells was reduced by MIAT knockdown. These findings indicate that MIAT functions by competing with critical RNAs to target miR-150-5p and activate zinc finger E-box binding homeobox 1 to modulate the function of osteosarcoma cells. Together, the present findings may contribute to the understanding of the pathogenesis of osteosarcoma.
\end{abstract}

\section{Introduction}

Osteosarcoma primarily impacts children, juveniles and adults from an early age (1). Although the worldwide incidence rate of this rare disease is only 3-4 cases per million, osteosarcoma is the most common form of bone cancer (2). Various chemotherapeutic and radiation treatments have been developed over the past two decades; however, the survival rate is still low. Almost $50 \%$ of patients succumb to pulmonary metastasis in the terminal stages of the disease (3). Elucidating the underlying molecular mechanisms of osteosarcoma metastasis is therefore a desirable research outcome.

The epithelial-mesenchymal transition (EMT) is involved in the process of invasion and metastasis in many cancers,

Correspondence to: Dr Wenbo Wang, Department of Orthopaedics, The First Affiliated Hospital of Harbin Medical University, 23 Youzheng Road, Harbin, Heilongjiang 150006, P.R. China

E-mail: 280798492@qq.com

Key words: osteosarcoma, epithelial-mesenchymal transition including osteosarcoma $(4,5)$. Zinc finger E-box binding homeobox 1 (ZEB1), is a key protein that functions to regulate the phenotype of EMT during cancer progression (6). ZEB1 may promote prostatic cancer, and overexpression of ZEB1 leads to the promotion of lung cancer cell metastasis $(7,8)$. Additionally, the overexpression of ZEB1 has been demonstrated to be associated with the development, carcinogenesis, invasion and metastasis of osteosarcoma (9).

Long non-coding RNAs (lncRNAs) are RNA molecules that have over 200 nucleotides and possess the potential for protein-coding (10). LncRNAs are involved in regulating cellular functions and the progression of various types of cancer (11). Several studies have demonstrated that lncRNAs serve a critical function in numerous cellular processes by competing with RNAs to regulate microRNAs (12-14). The myocardial infarction associated transcript (MIAT) may be expressed in postmitotic retinal precursor cells and mitotic progenitors (15). With regards to human malignancies, MIAT upregulation has been observed in conditions including neuroendocrine prostatic and gastric cancer $(16,17)$. However, the role of MIAT in the regulation of osteosarcoma remains unresolved.

\section{Materials and methods}

Tissue samples. Patients with osteosarcoma provided six tissue samples and were operated on at The First Affiliated Hospital of Harbin Medical University, China. Samples were snap frozen at $-80^{\circ} \mathrm{C}$ until RNA extraction. Written informed consent was obtained from all patients. The study was approved by the Research Ethics Committee at Harbin Medical University (Harbin, China).

Cell culture and transfection. The Chinese Cell Bank of the Chinese Academy of Sciences (Shanghai, China) provided the osteoblast cell lines hFOB (OB3) and osteosarcoma cell lines Saos-2 and MG-63. Dulbecco's modified Eagle's medium (DMEM; HyClone; GE Healthcare, Chicago, IL, USA) was used as cell medium at $37^{\circ} \mathrm{C}$, with $10 \%$ heat-inactivated fetal bovine serum (FBS; Biological Industries, Kibbutz Beit Haemek, Israel) and with $95 \%$ air and $5 \% \mathrm{CO}_{2}$. siRNAs targeting MIAT (forward, 5'-GGACGTTCACAACCACAC TG-3' and reverse, 5'-TCCCACTTTGGCATTCTAGG-3') were designed by Guangzhou RiboBio Co., Ltd. (Guangzhou, China). Knockdown and overexpression of miR-150-5p 
were obtained from Invitrogen (Thermo Fisher Scientific, Inc., Waltham, MA, USA). The sequences were as follows: Human miR-150-5p, 5'-UCUCCCAACCCUUGUACCAGU G-3' and 29-O-methyl modified miR-150 inhibitor, 5'-CAC UGGUACAAGGGUUGGGAGA-3'. Cell transfections were performed using X-tremeGENE siRNA Transfection Reagent (Roche Diagnostics, Indianapolis, IN, USA) according to the manufacturer's protocol. siNC (50 $\mu$ l DMEM was mixed with 20 pmol siNC; GCACCTTGAGTGAATGTCAGGGAC TCCCTGATGATGTGA; Guangzhou RiboBio Co., Ltd.) was defined as the negative control.

$R N A$ extraction and reverse transcription-quantitative polymerase chain reaction $(R T-q P C R)$. TRIzol ${ }^{\circledR}$ reagent (Life Technologies; Thermo Fisher Scientific, Inc.) was used to extract RNA according to the manufacturers protocol. Invitrogen (Thermo Fisher Scientific, Inc.) provided the PCR primers. A NanoDrop Spectrophotometer (NanoDrop Technologies, Wilmington, DE) was used to measure the concentration of extracted RNA. A TaqMan ${ }^{\circledR}$ miRNA reverse transcription kit (Applied Biosystems, Foster City, CA, USA) synthesized cDNA via RT in 5 ng of total RNA to find miR-150-5p levels. The $2^{-\Delta \Delta C q}$ method was used to determine the expression levels of miR-150-5p (18). MIAT expression was divided into high and low groups using RT-qPCR, using the median value as the cut-off to differentiate between the high and low groups. Bioinformatics analysis was used (MicroRNA, Starbase version 2.0) to determine the potential complementarity between MIAT and miRNAs.

In the synthesis kit of cDNA, RNA synthesized the cDNAs via specific gene primers. (Invitrogen; Thermo Fisher Scientific, Inc.) to determine ZEB1 mRNA expression. qPCR was performed using a SYBR Green Real-Time PCR Master Mix kit (Toyobo Life Science, Osaka, Japan) according to the manufacturer's protocol, and the ABI 7500 Sequence Detection System (Life Technologies; Thermo Fisher Scientific, Inc.). In a total reaction volume of $20 \mu \mathrm{l}$, amplification was performed with $1 \mu 1$ reverse primer, $1 \mu \mathrm{l}$ forward primer, $10 \mu \mathrm{l}$ SYBR Master Mix, $6 \mu 1$ diethyl pyrocarbonate and $2 \mu \mathrm{l}$ cDNA. The conditions for the reaction were as follows: $72^{\circ} \mathrm{C}$ for $45 \mathrm{sec}, 60^{\circ} \mathrm{C}$ for $15 \mathrm{sec}$ and 40 cycles of $95^{\circ} \mathrm{C}$ for $15 \mathrm{sec}$. The internal control was GAPDH. Expression levels of ZEB1 were determined in relation to GAPDH by the $2^{-\Delta \Delta C q}$ method (18). Table I presents the primer sequences.

Western blot analysis. Cells were washed using PBS three times at room temperature and lysed using radioimmunoprecipitation assay buffer with $1 \%$ protease inhibitor (Sigma-Aldrich; Merck KGaA, Darmstadt, Germany). The protein concentration was measured by Nanodrop. Proteins $(50 \mu \mathrm{g})$ were separated by SDS-PAGE $(10 \% \mathrm{gel})$. Protein transfer was then conducted onto nitrocellulose membranes. Blocking was performed using $0.1 \%$ Tween 20 and 5\% nonfat milk (BD Biosciences, Franklin Lakes, NJ, USA) in TBS for $2 \mathrm{~h}$ at room temperature. Samples were incubated with the proper primary antibodies (cat. no. ab203829; 1:1,000; rabbit; Abcam, Cambridge, MA, USA) at $4^{\circ} \mathrm{C}$ overnight with gentle agitation followed by staining with the fluorochrome-labeled secondary antibody (cat. no. A10235; 1:8,000; rabbit anti-mouse; Alexa Fluor 800; LI-COR Biosciences, Lincoln, NE, USA) at room temperature for $1 \mathrm{~h}$. The Odyssey fluorescent scanning system (LI-COR Biosciences) detected immunoreactivity and Image Studio software 4.0 (LI-COR Biosciences) was used to examine captured images. The loading control was $\beta$-actin.

Cell proliferation assay. The cell counting kit-8 kits (CCK-8) were used according to the manufacturer's instructions. MG63 and Saos-2 cells were seeded in 96-well plates at $1 \times 10^{4}$ cells/well for $24 \mathrm{~h}$. CCK-8 solution $(10 \mu \mathrm{l})$ was added to each well, and the cells were incubated at $37^{\circ} \mathrm{C}$ for $2 \mathrm{~h}$. Absorbance at $450 \mathrm{~nm}$ was measured using a microplate reader. The assay was conducted in triplicate.

Wound healing assays. Osteosarcoma cells (MG63 and Saos-2 cells) were seeded in six-well plates and allowed to reach $80-90 \%$ confluence. A wound line was drawn across the surface of the plates using a $200-\mu l$ sterile plastic tip. PBS was used to wash the plates. Images were captured $24 \mathrm{~h}$ post wound infliction. Each test was conducted in triplicate.

Transwell assays. Transwell filters $(8 \mu \mathrm{m}$ pore size; BD Biosciences) were placed on a 24-well plate containing DMEM/F12 (Hyclone: GE Healthcare). The medium in the upper membrane was serum free, and in the lower chamber contained 10\% FBS (Biological Industries, Beit-Haemek, Israel).

MG63 and Saos-2 cells were suspended in DMEM/F12 at a cell density of $2.5 \times 10^{5}$ cells $/ \mathrm{ml}$ for $24 \mathrm{~h}$. After $24 \mathrm{~h}$, cells present on the top of the membrane were cleared using a cotton swab. Cells present on the bottom portion of the membrane were fixed using 4\% paraformaldehyde in PBS for $10 \mathrm{~min}$ and stained using crystal violet at room temperature for $15 \mathrm{~min}$. Cell invasion was quantified as the average number of cells from 3 inserts present on the bottom portion of the membrane.

Statistical analysis. All data are expressed as the mean \pm standard error of the mean. SPSS software (version 13.0; SPSS, Inc., Chicago, IL, USA) was used to analyze all data. Statistical comparison of two groups was performed using a Student's t-test. One-way analysis of variance was also used to compare $\geq 2$ groups. $\mathrm{P}<0.05$ was considered to indicate a statistically significant difference.

\section{Results}

MIAT levels are elevated in osteosarcoma. The expression levels of the lncRNA MIAT 6 were investigated in groups of osteosarcoma tissues using RT-qPCR. MIAT levels were decreased in adjacent non-tumor tissues compared with osteosarcoma tissues (Fig. 1A). The lncRNA MIAT expression levels were also investigated in MG63, Saos-2 and OB3 cells and it was identified that MIAT expression was lower in the OB3 cell line than in Saos-2 and MG63 cells (Fig. 1B). These results demonstrate that MIAT levels are potentially associated with osteosarcoma.

MIAT knockdown inhibits the proliferation and invasion of osteosarcoma cells. The function of MIAT in the proliferation and invasion of osteosarcoma cells was examined. Transfection of MG63 and Saos-2 cells with MIAT siRNAs 
Table I. Primers used for RT-PCR.

Name

Sequence (5'-3')

Length (bp)

GAPDH forward

GAPDH reverse

miR-150-5p forward

miR-150-5p reverse

ZEB1 forward

ZEB1 reverse

U6 forward

U6 reverse

$\begin{array}{ll}\text { AAGAAGGTGGTGAAGCAGGC } & 20 \\ \text { TCCACCACCCTGTTGCTGTA } & 20 \\ \text { GTCTCCCAACCCTTGTAC } & 18 \\ \text { TATCCAGTGCGTGTCGTG } & 18 \\ \text { FGCCAATAAGCAAACGATTCTG } & 22 \\ \text { TTTGGCTGGATCACTTTCAAG } & 21 \\ \text { CTCGCTTCGGCAGCACATATACT } & 23 \\ \text { ACGCTTCACGAATTTGCGTGTC } & 22\end{array}$

ZEB1, zinc finger E-box binding homeobox 1; RT-PCR, reverse transcription-polymerase chain reaction.
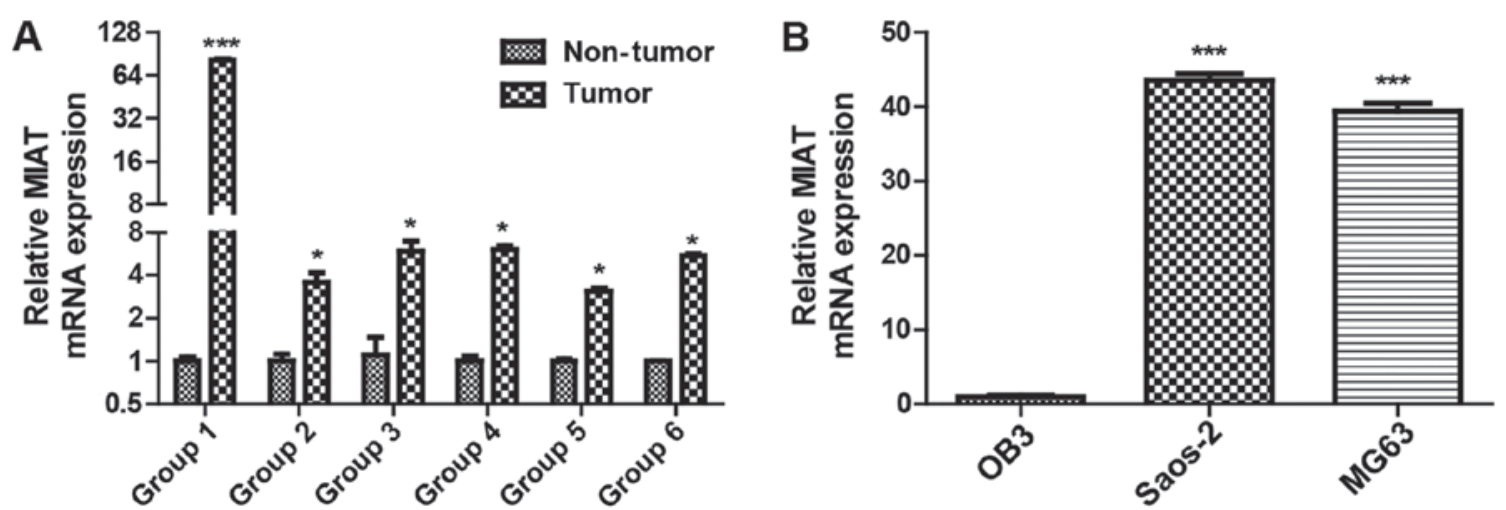

Figure 1. mRNA expression levels of MIAT are elevated in osteosarcoma. (A) MIAT mRNA expression levels in six groups of osteosarcoma tissues are significantly higher than those in non-tumor tissues as determined by reverse transcription-quantitative polymerase chain reaction. (B) MIAT mRNA expression levels in OB3, MG63 and Saos-2 cells. Data are expressed as the mean \pm the standard error of the mean. $\mathrm{n}=3$. ${ }^{*} \mathrm{P}<0.05$ and ${ }^{* * * *} \mathrm{P}<0.001$ compared with the control group. MIAT, myocardial infarction associated transcript.

was demonstrated to lower MIAT expression in comparison to control cells (Fig. 2A). CCK8 results indicated that knockdown of MIAT reduced MG63 and Saos-2 cell proliferation compared to cells transfected with siRNAs (Fig. 2B). MIAT knockdown also limited the proliferation and invasion of MG63 and Saos-2 cells (Fig. 2C and D) compared to cells transfected with siRNAs. Taken together, these data reveal that MIAT may promote proliferation and invasion of osteosarcoma cells in vitro.

MIAT promotes ZEB1 expression in osteosarcoma. ZEB1 is important for the proliferation and invasion of osteosarcoma cells (19). Thus, it was investigated whether MIAT affects ZEB1 expression in osteosarcoma cells. First, the association between MIAT and the ZEB1 expression levels was evaluated in 6 samples of tumor-adjacent tissue and osteosarcoma samples by western blotting and RT-qPCR.

It was identified that ZEB1 expression was notably increased in the high MIAT osteosarcoma tissue group compared with that in the low MIAT group (Fig. 3A and B). It was additionally noted that ZEB1 expression levels were decreased in OB3 cells compared with MG63 and Saos-2 cells (Fig. 3C and D). Furthermore, ZEB1 expression levels were evaluated in Saos-2 and MG63 cells transfected with MIAT siRNAs or siNC (negative control) and it was identified that MIAT knockdown by siRNAs resulted in lower ZEB1 expression compared to cells transfected with control siRNAs (Fig. 3E and F).

miR-150-5p is a downstream target of MIAT. To identify the potential downstream miRNA targets of MIAT and its interactions in osteosarcoma, bioinformatics analysis was used (MicroRNA, Starbase version 2.0) to determine the potential complementarity between MIAT and miRNAs. Bioinformatics predictions revealed that the MIAT sequence has four putative miRNA binding sites, including sites for miR-29a-3p, miR-29b-3p, miR-29c-3p, and miR-150-5p-5p. Yan et al (20) previously reported that miR-150-5p-5p focuses on MIAT in endothelial cells, and another study revealed that miR-150-5p suppresses ZEB1 in epithelial ovarian cancer (21). It may be observed that miR-150-5p levels were higher in adjacent non-tumor tissues than in osteosarcoma tissues (Fig. 4A) and lower in MG63 and Saos-2 cells than OB3 cells (Fig. 4B).

To determine whether miR-150-5p targets MIAT, miR-150-5p expression in Saos-2 and MG63 cells transfected with MIAT-siRNA or siNC was examined. The results revealed that miR-150-5p expression was visibly elevated in MG63 and Saos-2 cells transfected with MIAT-siRNA compared to control siRNA (Fig. 5A and B). Rescue experiments were subsequently performed by transfecting miR-150-5p in Saos-2 


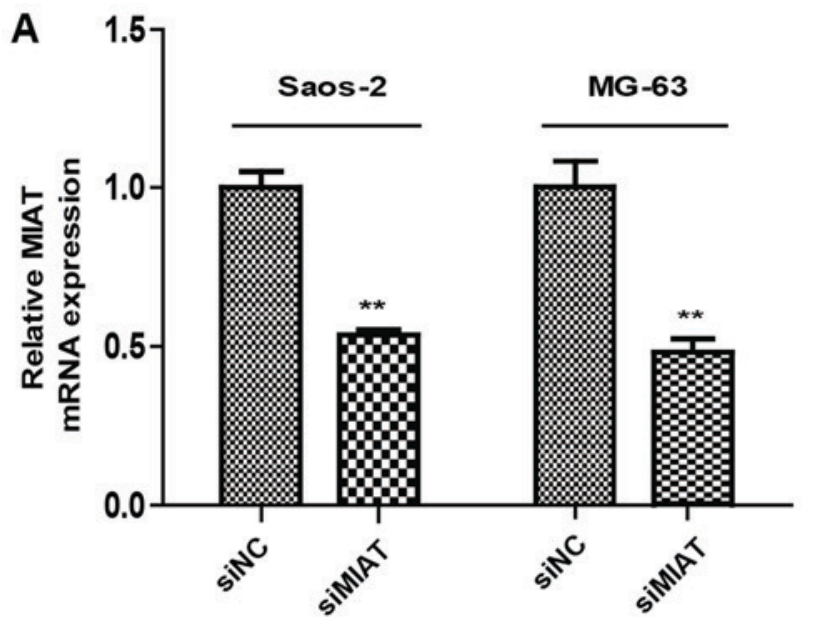

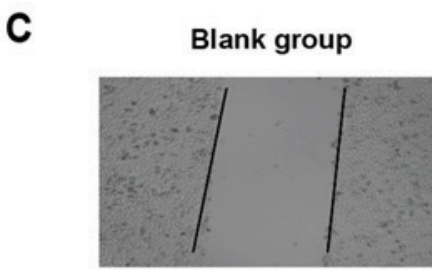

Saos-2
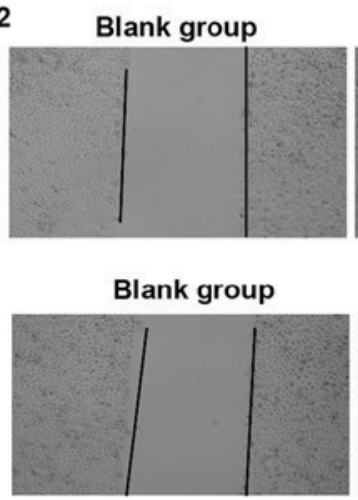

MG-63

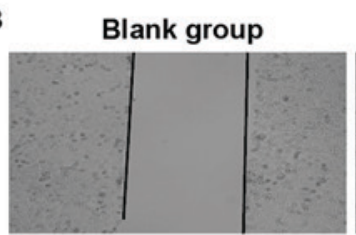

siNC

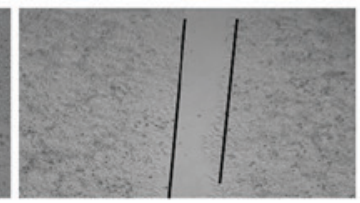

SIMIAT

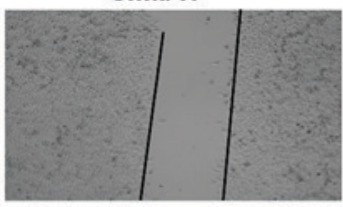

siNC

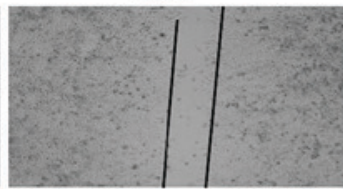

SIMIAT

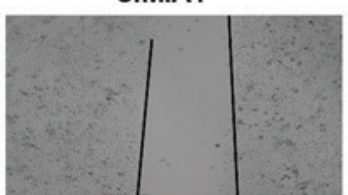

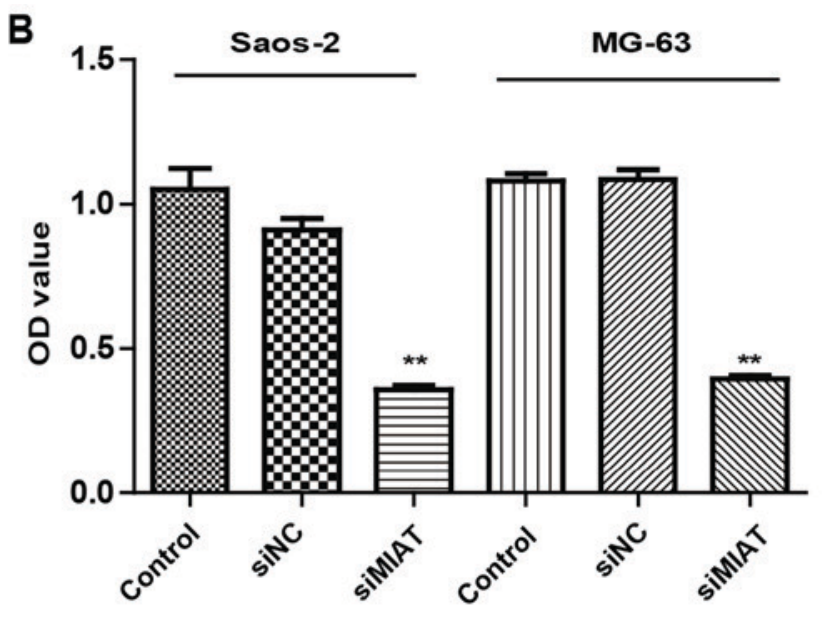

B

D
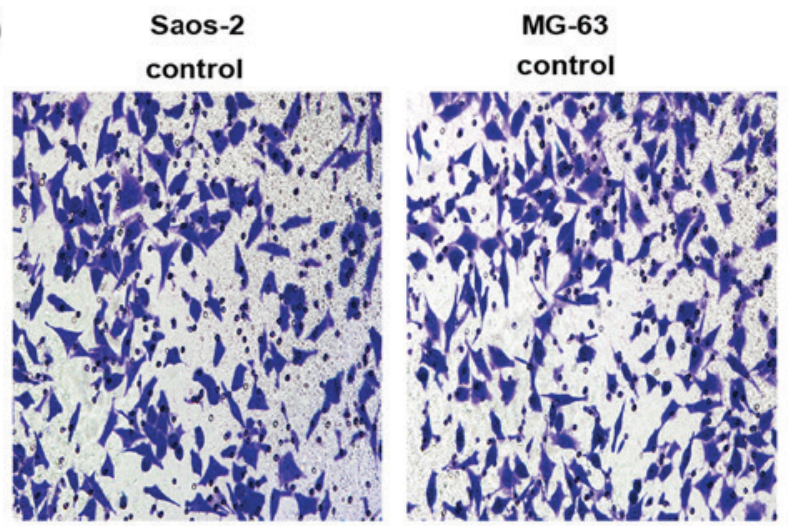

SIMIAT

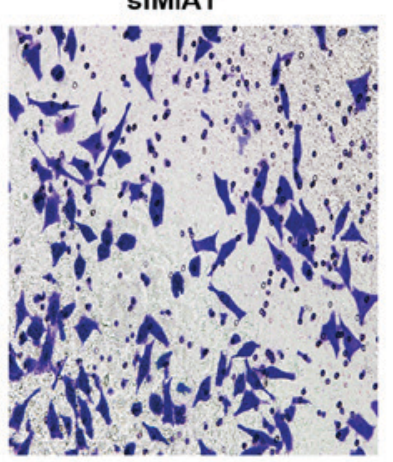

SIMIAT

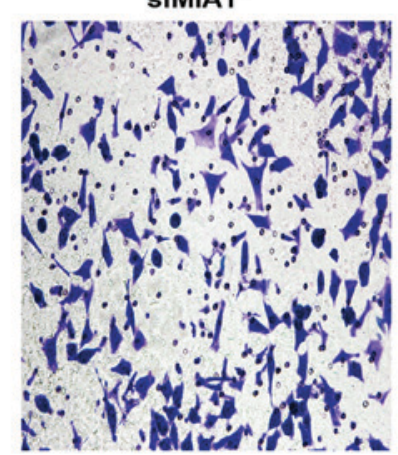

Figure 2. MIAT knockdown inhibits the proliferation and invasion of osteosarcoma cells. (A) Expression levels of MIAT in Saos-2 and MG-63 osteosarcoma cells following transfection with MIAT siRNAs. (B) Cell proliferation determined by cell counting kit 8 . Knockdown of MIAT also reduced cell proliferation detected by $(\mathrm{C})$ wound healing assays and (D) invasion assays. Data are expressed as the mean \pm the standard error of the mean. $n=3 .{ }^{* *} \mathrm{P}<0.01$ and ${ }^{* * * *} \mathrm{P}<0.001$ compared with the control group. MIAT, myocardial infarction associated transcript.

and MG63 cells. Overexpressing miR-150-5p led to an increase in ZEB1 (Fig. 4C and D). Additionally, inhibiting MIAT limited ZEB1 levels by inhibiting miR-150-5p (Fig. 5A and B). Together, the results demonstrate the importance of MIAT in regulating ZEB1, by controlling miR-150-5p.

miR-150-5p reverses the effects of MIAT in osteosarcoma cells. These results demonstrated that miR-150-5p is a downstream target of MIAT. However, the function of miR-150-5p in the MIAT-mediated influence on osteosarcomacells remains unclear. To determine whether MIAT may promote proliferation and invasion of osteosarcoma cells via the MIAT-miR-150-5p-ZEB1 axis, further experiments were carried out. RT-qPCR and western blotting revealed that reduced ZEB1 expression by inhibition of MIAT could be largely reversed by AMO-miR-150-5p (Fig. 5). Together, these results suggest that miR-150-5p was able to change the function of MIAT in osteosarcoma cells and that MIAT was able to promote the proliferation and invasion of osteosarcoma cells via the MIAT-miR-150-5p-ZEB1 axis.

\section{Discussion}

Osteosarcoma has been considered a common type of basic malignancy of bone and is derived from the progenitor mesenchymal cells of bone-forming cells (22). The morbidity associated with osteosarcoma is high since early diagnosis is difficult, and 
A

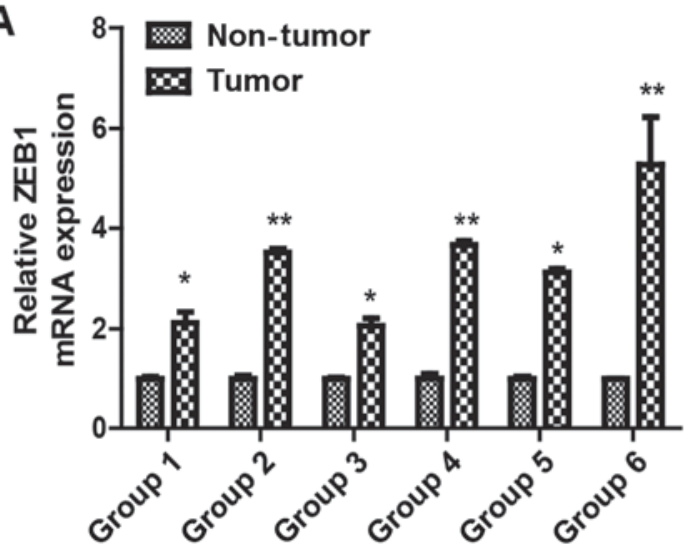

C

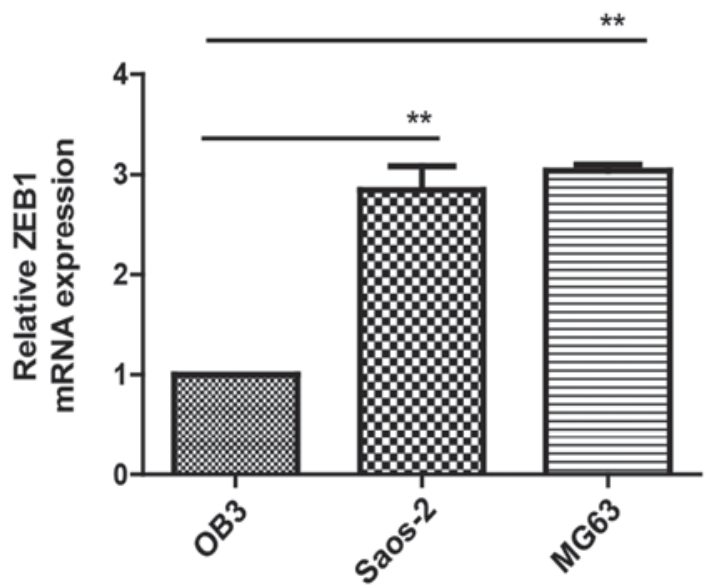

E

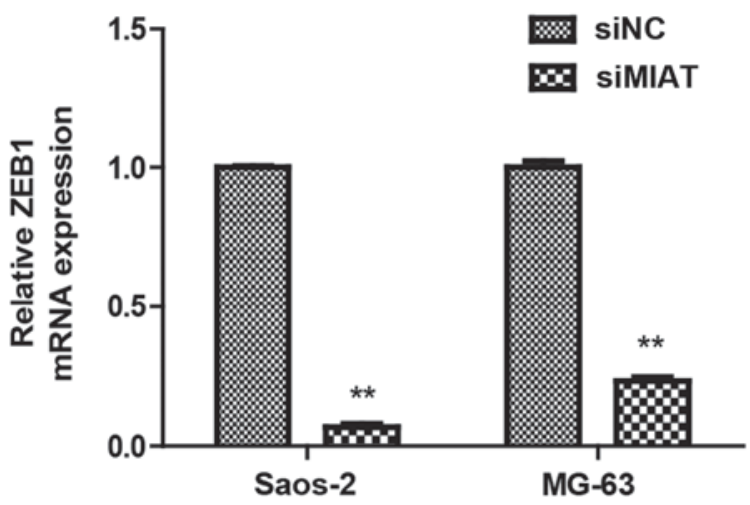

B

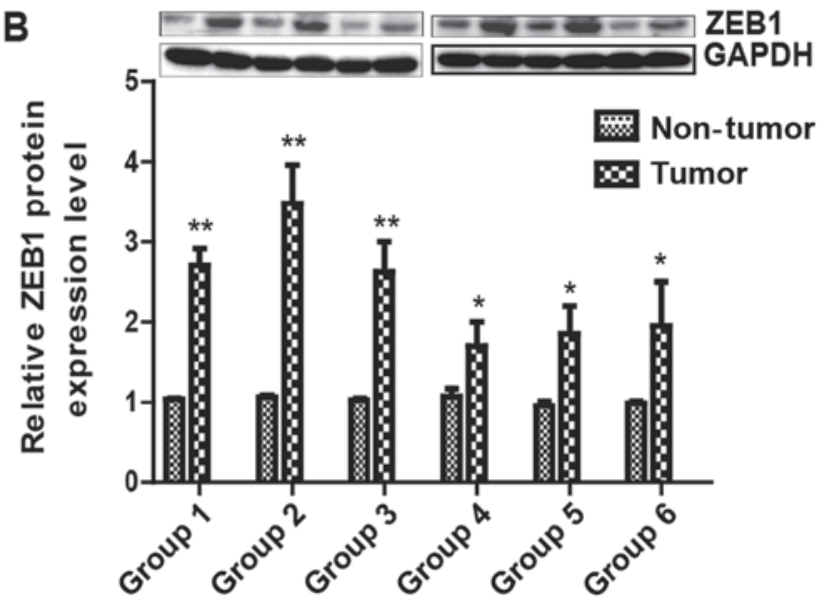

D

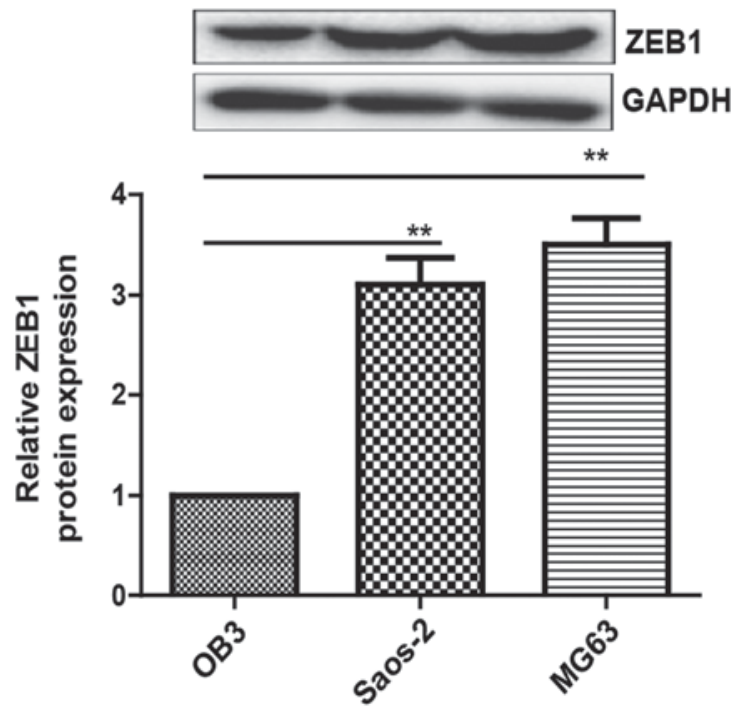

F

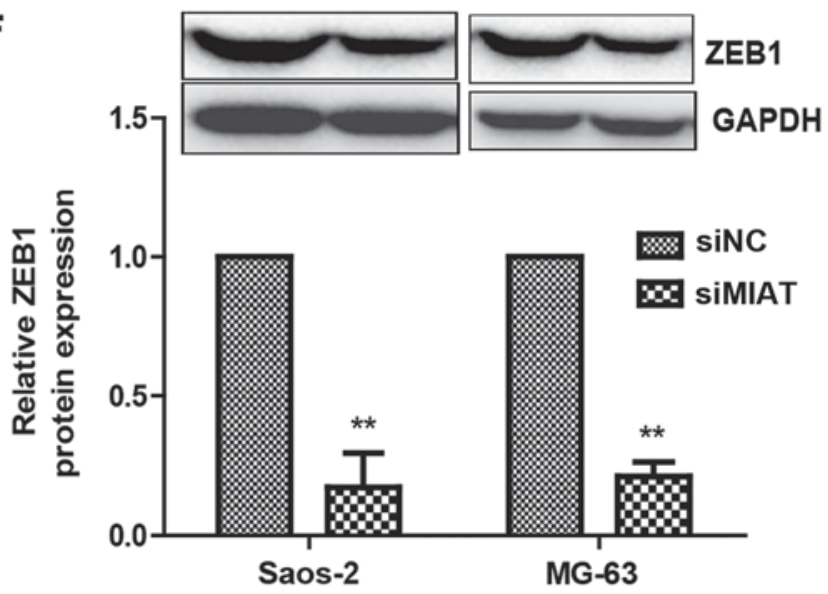

Figure 3. MIAT promotes ZEB1 expression in osteosarcoma. ZEB1 (A) mRNA and (B) protein expression levels are notably increased in the high MIAT osteosarcoma tissues groups compared to that in the low groups. The (C) mRNA and (D) protein expression levels of ZEB1are elevated in Saos-2 and MG-63 osteosarcoma cells compared with OB3 osteosarcoma cells. The (E) mRNA and (F) protein expression levels of ZEB1, MG63 and Saos-2 cells transfected with MIAT siRNAs had lower ZEB1 expression. Data are expressed as the mean \pm the standard error of the mean. $\mathrm{n}=3$. ${ }^{*} \mathrm{P}<0.05$ and ${ }^{* *} \mathrm{P}<0.01$ compared with the control group. MIAT, myocardial infarction associated transcript; ZEB1, zinc finger E-box binding homeobox 1.

therapeutic solutions to osteosarcoma are lacking. Therefore, it is important to identify new molecules associated with developing osteosarcomas and develop novel targeted therapy strategies.

Previous studies have demonstrated the role of IncRNAs (23) and the molecular mechanisms through which lncRNAs affect human tumors (24-26). However, the mechanism involving the lncRNA MIAT in osteosarcoma has remained unknown. This research shows that highly overexpressed MIAT in cell lines and osteosarcoma tissues may have a monogenic role. 

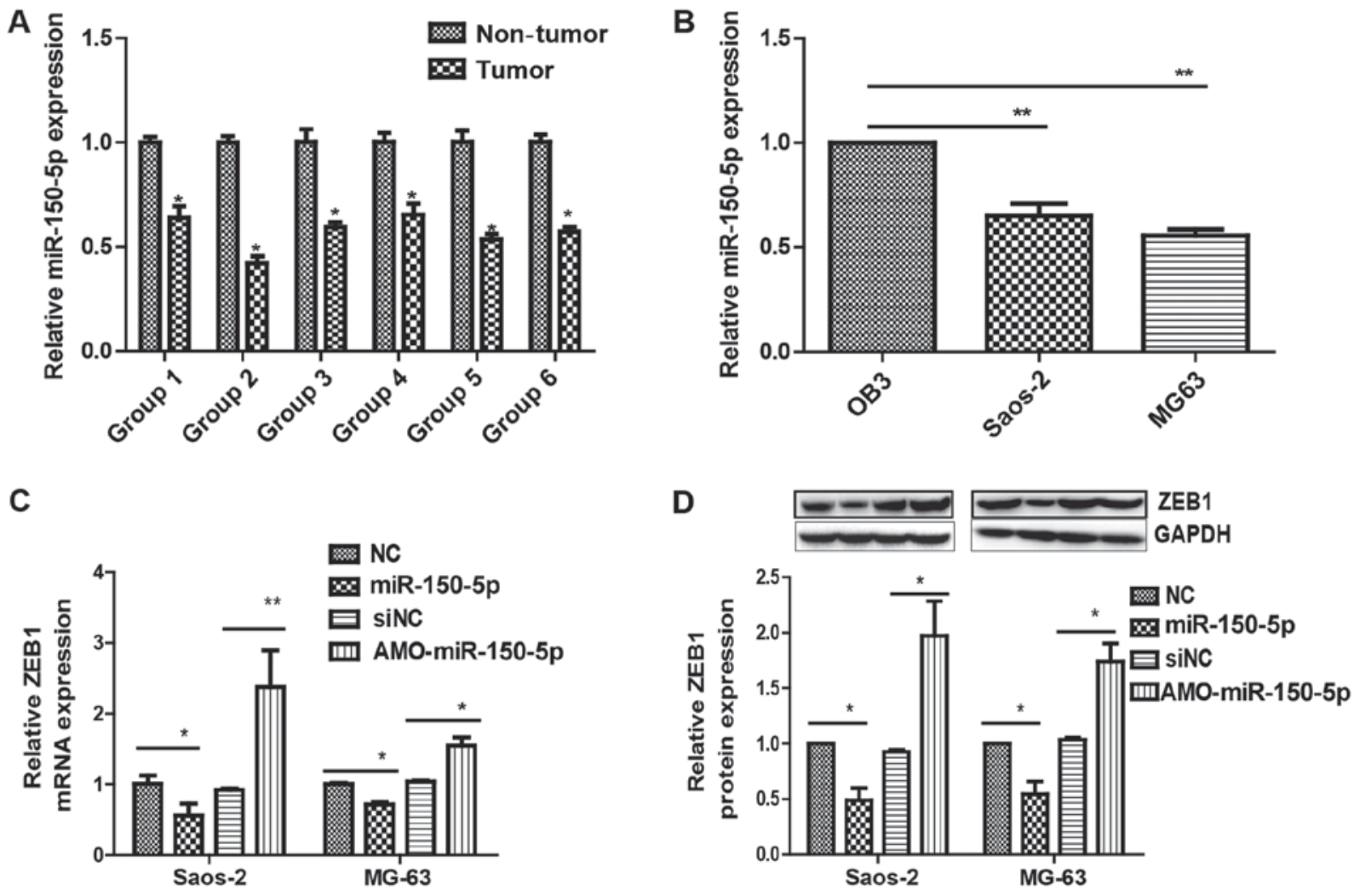

Figure 4. ZEB1 is a downstream target of miR-150-5p. (A) miR-150-5p levels were decreased in six groups of osteosarcoma tissues compared to adjacent non-tumor tissues. (B) miR-150-5p levels were decreased in Saos-2 and MG-63 osteosarcoma cells compared to OB3 osteosarcoma cells. ZEB1 (C) mRNA and (D) protein levels were decreased when Saos-2 and MG-63 osteosarcoma cells were transfected with miR-150-5p and increased when transfected with miR-150-5p inhibitor. Data are expressed as the mean \pm the standard error of the mean. $n=3 .{ }^{*} \mathrm{P}<0.05,{ }^{* *} \mathrm{P}<0.01$ compared with the control group. MIAT, myocardial infarction associated transcript; ZEB1, zinc finger E-box binding homeobox 1.
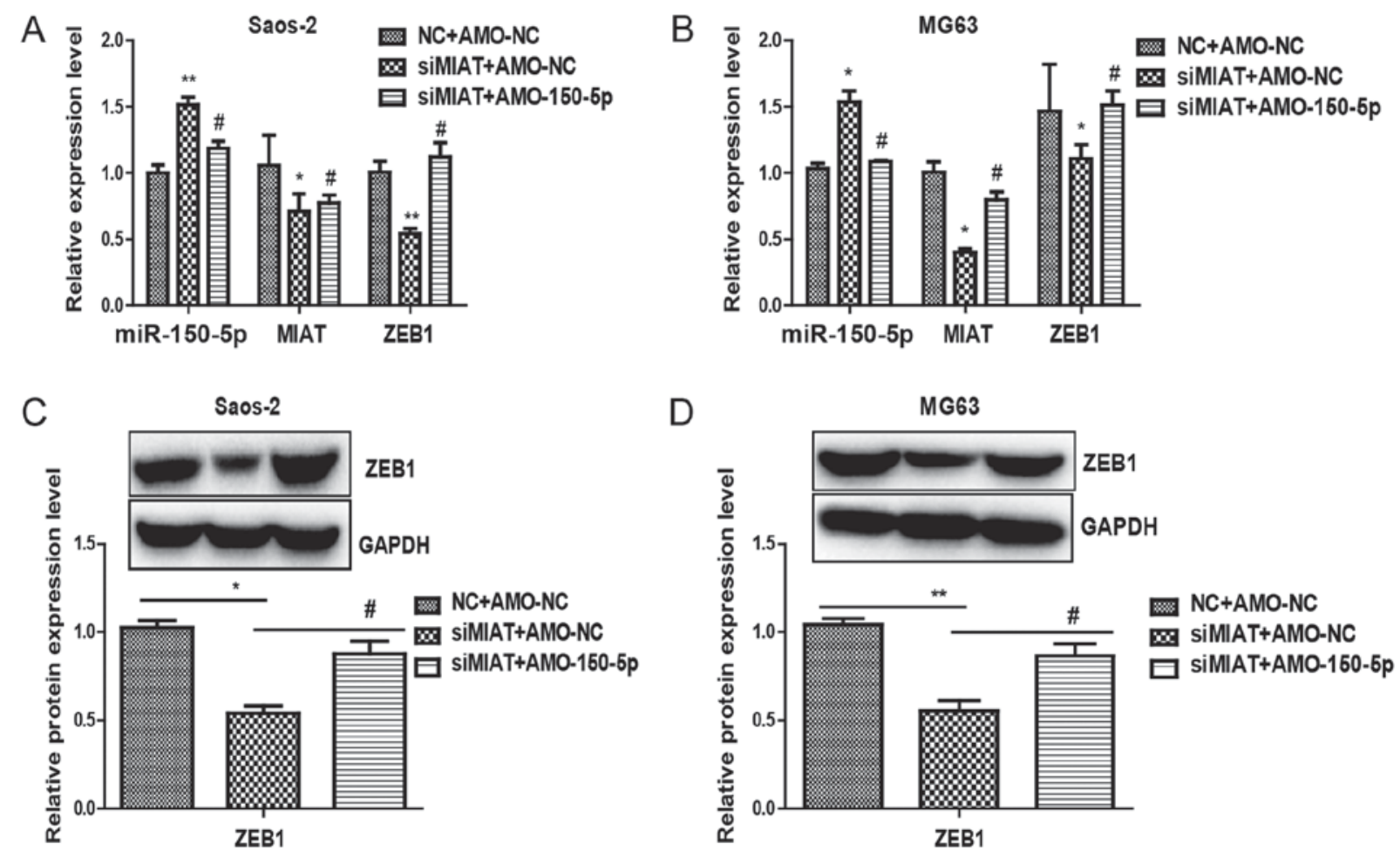

Figure 5. MIAT regulates ZEB1 levels through miR-150-5p. miR-150-5p expression was visibly elevated in (A) Saos-2 and (B) MG63 cells transfected with MIAT-siRNA. ZEB1 mRNA expression levels are reduced by inhibition of MIAT and reversed by a miR-150-5p inhibitor. ZEB1 protein expression levels are in accordance with mRNA expression levels in (C) Saos-2 and (D) MG63 cells. Data are expressed as the mean \pm the standard error of the mean. $n=3$. ${ }^{*} \mathrm{P}<0.05$, ${ }^{* *} \mathrm{P}<0.01$ compared with the control group, ${ }^{*} \mathrm{P}<0.05$ vs. siMIAT groups. MIAT, myocardial infarction associated transcript; ZEB1, zinc finger E-box binding homeobox 1 . 
miR-150-5p was first considered as the main miRNA in immune and hematopoietic cells (27). Research has recently shown that specific cellular functions in diverse tumors also involve miR-150-5p (28-30). A previous study demonstrated that expression of miR-150-5p was decreased in osteosarcoma cells compared to analogous human normal osteoblasts and cells from normal tissues (31).

MIAT was identified as a target of miR-150-5p with both MIAT and miR-150-5p having an inhibitory effect. These results demonstrate that MIAT may improve tumor progression in osteosarcoma since it can inhibit miR-150-5p and activate ZEB1. The present study demonstrated that in osteosarcoma tissues, MIAT expression was increased compared with the adjacent normal tissues. The present study also identifies that MIAT is important in the development and progression of osteosarcoma. However, the mechanisms associated with MIAT-mediated gene expression in tumorigenesis need to be clarified.

Research has revealed that MIAT acts as a molecular sponge by managing microRNAs in the progression of breast cancer (32). It has been reported that during specific cellular processes, IncRNAs are able to compete with endogenous RNAs to manage microRNAs (33). MIAT may therefore be considered as an endogenous miRNA which controls miR-150-5p-5p and manages its role. Although several potential miRNA binding partners were identified, the present study focused on miR-150-5p, as it has been demonstrated to be important in numerous cancers, including lung (34) and liver cancer (35). The present study revealed that miR-150-5p levels were decreased in the osteosarcoma cell lines MG63 and Saos-2 cells. An opposing association was observed between miR-150-5p and MIAT levels in Saos-2 and MG63 cells, suggesting that there may be an association between miR-150-5p and MIAT to control osteosarcoma cell proliferation and invasion. In addition, miR-150-5p expression decreased ZEB1 levels in Saos-2 cells and MG63. Furthermore, the present data suggested that the MIAT sequence had miR-150-5p sites for binding and implied that miR-150-5p reduced MIAT level by directly binding MIAT.ZEB1 is a master regulator of the EMT phenotype within the progression of cancer (36). The present study demonstrates that by the miR-150-5p/ZEB1 pathway, MIAT may induce EMT phenotype in osteosarcoma cells.

To conclude, the present study revealed that MIAT may be a biomarker for patients with osteosarcoma. It is hypothesized that the MIAT-miR-150-5p-ZEB1 axis may be a potential therapeutic target in osteosarcoma.

\section{Acknowledgements}

Not applicable.

\section{Funding}

This study was financially supported by Heilongjiang provincial academy of medical sciences (grant no. 201618).

\section{Availability of data and materials}

The analyzed datasets generated during the study are available from the corresponding author on reasonable request.

\section{Authors' contributions}

HJ performed the flow cytometric analysis and drafted the manuscript. XJ performed cell culture and viral preparation experiments. WC and FD contributed to statistical analyses. ZY, YL and WW designed the study.

\section{Ethics approval and consent to participate}

Written informed consent was obtained from all patients. The study was approved by the Research Ethics Committee at Harbin Medical University.

\section{Patient consent for publication}

Not applicable.

\section{Competing interests}

The authors declare that they have no competing interests.

\section{References}

1. Liu C and Lin J: Long noncoding RNA ZEB1-AS1 acts as an oncogene in osteosarcoma by epigenetically activating ZEB1. Am J Transl Res 8: 4095-4105, 2016.

2. Mirabello L, Troisi RJ and Savage SA: Osteosarcoma incidence and survival rates from 1973 to 2004: Data from the surveillance, epidemiology, and end results program. Cancer 115: 1531-1543, 2009.

3. Daw NC, Chou AJ, Jaffe N, Rao BN, Billups CA, Rodriguez-Galindo C, Meyers PA and Huh WW: Recurrent osteosarcoma with a single pulmonary metastasis: A multiinstitutional review. Br J Cancer 112: 278-282, 2012.

4. Yu L, Liu S, Guo W, Zhang C, Zhang B, Yan H and Wu Z: hTERT promoter activity identifies osteosarcoma cells with increased EMT characteristics. Oncol Lett 7: 239-244, 2014.

5. Sung JY, Park SY, Kim JH, Kang HG, Yoon JH, Na YS, Kim YN and Park BK: Interferon consensus sequence-binding protein (ICSBP) promotes epithelial-to-mesenchymal transition (EMT)-like phenomena, cell-motility, and invasion via TGF- $\beta$ signaling in U2OS cells. Cell Death Dis 5: e1224, 2014.

6. Al-Khalaf $\mathrm{HH}$ and Aboussekhra A: MicroRNA-141 and microRNA-146b-5p inhibit the prometastatic mesenchymal characteristics through the RNA-binding protein AUF1 targeting the transcription factor ZEB1 and the protein kinase AKT. J Biol Chem 289: 31433-31447, 2014

7. Putzke AP, Ventura AP, Bailey AM, Akture C, Opoku-Ansah J, Celiktaş M, Hwang MS, Darling DS, Coleman IM, Nelson PS, et al: Metastatic progression of prostate cancer and e-cadherin regulation by zeb1 and SRC family kinases. Am J Pathol 179: 400-410, 2011.

8. Roy BC, Kohno T, Iwakawa R, Moriguchi T, Kiyono T, Morishita K, Sanchez-Cespedes M, Akiyama T and Yokota J: Involvement of LKB1 in epithelial-mesenchymal transition (EMT) of human lung cancer cells. Lung Cancer 70: 136-145, 2010.

9. Shen A, Zhang Y, Yang H, Xu R and Huang G: Overexpression of ZEB1 relates to metastasis and invasion in osteosarcoma. J Surg Oncol 105: 830-834, 2012.

10. Nagano T and Fraser P: No-nonsense functions for long noncoding RNAs. Cell 145: 178-181, 2011.

11. Chen LL and Zhao JC: Functional analysis of long noncoding RNAs in development and disease. Adv Exp Med Biol 825: 129-158, 2014.

12. Zhu HY, Bai WD, Li C, Zheng Z, Guan H, Liu JQ, Yang XK, Han SC, Gao JX, Wang HT and Hu DH: Knockdown of lncRNA-ATB suppresses autocrine secretion of TGF- $\beta 2$ by targeting ZNF217 via miR-200c in keloid fibroblasts. Sci Rep 6: $24728,2016$.

13. Wu X, He X, Li S, Xu X, Chen X and Zhu H: Long non-coding RNA ucoo $2 \mathrm{kmd} .1$ regulates CD44-dependent cell growth by competing for miR-211-3p in colorectal cancer. PLoS One 11: e0151287, 2016. 
14. Wang GQ, Wang Y, Xiong Y, Chen XC, Ma ML, Cai R, Gao Y, Sun YM, Yang GS and Pang WJ: Sirt1 AS IncRNA interacts with its mRNA to inhibit muscle formation by attenuating function of miR-34a. Sci Rep 6: 21865, 2016.

15. Ishii N, Ozaki K, Sato H, Mizuno H, Saito S, Takahashi A, Miyamoto Y,Ikegawa S, Kamatani N, Hori M, et al: Identification of a novel non-coding RNA, MIAT, that confers risk of myocardial infarction. J Hum Genet 51: 1087-1099, 2006.

16. Crea F, Venalainen E, Ci X, Cheng H, Pikor L, Parolia A, Xue H, Nur Saidy NR, Lin D, Lam W, et al: The role of epigenetics and long noncoding RNA MIAT in neuroendocrine prostate cancer. Epigenomics 8: 721-731, 2016.

17. Sha M, Lin M, Wang J, Ye J, Xu J, Xu N and Huang J: Long non-coding RNA MIAT promotes gastric cancer growth and metastasis through regulation of miR-141/DDX5 pathway. J Exp Clin Cancer Res 37: 58, 2018.

18. Livak KJ and Schmittgen TD: Analysis of relative gene expression data using real-time quantitative PCR and the 2(-Delta Delta C(T)) method. Methods 25: 402-408, 2001

19. Yan H, Zhang B, Fang C and Chen L: miR-340 alleviates chemoresistance of osteosarcoma cells by targeting ZEB1. Anticancer Drugs 29: 440-448, 2018.

20. Yan B, Yao J, Liu JY, Li XM, Wang XQ, Li YJ, Tao ZF, Song YC, Chen $Q$ and Jiang Q: LncRNA-MIAT regulates microvascular dysfunction by functioning as a competing endogenous RNA. Circ Res 116: 1143-1156, 2015.

21. Jin M, Yang Z, Ye W, Xu H and Hua X: MicroRNA-150 predicts a favorable prognosis in patients with epithelial ovarian cancer, and inhibits cell invasion and metastasis by suppressing transcriptional repressor ZEB1. PLoS One 9: e103965, 2014.

22. Lulla RR, Costa FF, Bischof JM, Chou PM, de F Bonaldo M, Vanin EF and Soares MB: Identification of differentially expressed MicroRNAs in osteosarcoma. Sarcoma 2011: 732690, 2011.

23. Orom UA, Derrien T, Beringer M, Gumireddy K, Gardini A Bussotti G, Lai F, Zytnicki M, Notredame C, Huang Q, et al: Long noncoding RNAs with enhancer-like function in human cells. Cell 143: 46-58, 2010.

24. Barnhill LM, Williams RT, Cohen O, Kim Y, Batova A, Mielke JA, Messer K, Pu M, Bao L, Yu AL and Diccianni MB: High expression of CAI2, a 9p21-embedded long noncoding RNA, contributes to advanced-stage neuroblastoma. Cancer Res 74: 3753-3763, 2014.

25. Nie FQ,Zhu Q, Xu TP, Zou YF, Xie M, Sun M, Xia R and Lu KH Long non-coding RNA MVIH indicates a poor prognosis for non-small cell lung cancer and promotes cell proliferation and invasion. Tumour Biol 35: 7587-7594, 2014.
26. Zhen L, Yun-Hui L, Hong-Yu D, Jun M and Yi-Long Y: Long noncoding RNA NEAT1 promotes glioma pathogenesis by regulating miR-449b-5p/c-Met axis. Tumour Biol 37: 673-683, 2016.

27. He Y, Jiang $X$ and Chen J: The role of miR-150-5p in normal and malignant hematopoiesis. Oncogene 33: 3887-3893, 2014.

28. Wang X, Ren Y, Wang Z, Xiong X, Han S, Pan W, Chen H, Zhou L, Zhou C, Yuan Q and Yang M: Down-regulation of 5S rRNA by miR-150-5p and miR-383 enhances c-Myc-rpL11 interaction and inhibits proliferation of esophageal squamous carcinoma cells. FEBS Lett 589: 3989-3997, 2015.

29. Liu DZ, Zhang HY, Long XL, Zou SL, Zhang XY, Han GY and Cui ZG: MIR-150-5P promotes prostate cancer stem cell development via suppressing p27Kip1. Eur Rev Med Pharmacol Sci 19: 4344-4352, 2015.

30. Li J, Hu L, Tian C, Lu F, Wu J and Liu L: microRNA-150 promotes cervical cancer cell growth and survival by targeting FOXO4. BMC Mol Biol 16: 24, 2015.

31. Qu Y, Pan S, Kang M, Dong R and Zhao J: MicroRNA-150 functions as a tumor suppressor in osteosarcoma by targeting IGF2BP1. Tumor Biol 37: 5275-5284, 2016

32. Luan T, Zhang X, Wang S, Wang S, Song Y, Zhou S, Lin J, An W, Yuan W, Yang Y, et al: Long non-coding RNA MIAT promotes breast cancer progression and functions as ceRNA to regulate DUSP7 expression by sponging miR-155-5p. Oncotarget 8 : 76153-76164, 2017

33. Mercer TR, Dinger ME and Mattick JS: Long non-coding RNAs: Insights into functions. Nat Rev Genet 10: 155-159, 2009.

34. Cao M, Hou D, Liang H, Gong F, Wang Y, Yan X, Jiang X, Wang C, Zhang J, Zen K, et al: miR-150-5p promotes the proliferation and proliferation of lung cancer cells by targeting SRC kinase signalling inhibitor 1. Eur J Cancer 50: 1013-1024, 2014.

35. Li T, Xie J, Shen C, Cheng D, Shi Y, Wu Z, Zhan Q, Deng X, Chen $\mathrm{H}$, Shen B, et al: miR-150-5p-5p inhibits hepatoma cell proliferation and invasion by targeting MMP14. PLoS One 9: e115577, 2014

36. Preca BT, Bajdak K, Mock K, Sundararajan V, Pfannstiel J, Maurer J, Wellner U, Hopt UT, Brummer T, Brabletz S, et al: A self-enforcing CD44S/ZEB1 feedback loop maintains EMT and stemness properties in cancer cells. Int J Cancer 137: 2566-2577, 2015. 